\title{
水/醇溶聚合物界面材料在聚合物光电器件中的应用及性能研究
}

\author{
张凯管星黄飞* 曹镛 \\ (华南理工大学 发光材料与器件国家重点实验室＼cjkstart高分子光电材料与器件研究所 广州 510640)
}

\begin{abstract}
摘要 系统研究了系列不同共轭与非共轭水/醇溶聚合物作为界面修饰材料在聚合物发光二极管和聚合物太阳电池中 的应用及结构性能关系. 研究了界面层厚度, 器件金属电极功函对材料界面修饰性能的影响. 在此基础上, 系统对比 研究了共轭与非共轭水/醇溶聚合物界面材料在不同聚合物光电器件中界面修饰性能的差别. 内建电势测试与器件研 究结果表明, 在聚合物发光二极管中, 共轭材料表现出明显优于非共轭材料的性能, 特别是在超高功函数的金属金电 极器件中, 共轭的水/醇溶聚合物材料依然表现出很好的电子注入/传输性能; 在聚合物太阳电池中, 共轭材料的界面修 饰性能也优于非共轭类界面修饰材料.
\end{abstract}

关键词＼cjkstart聚合物发光二极管; 聚合物太阳电池; 界面材料; 水/醇溶聚合物

\section{Performance Study of Water/Alcohol Soluble Polymer Interface Materials in Polymer Optoelectronic Devices}

\author{
Zhang, Kai Guan, Xing Huang, Fei* Cao, Yong \\ (State Key Laboratory of Luminescent Materials and Devices, Institute of Polymer Optoelectronic Materials and Devices, \\ South China University of Technology, Guangzhou 510640)
}

\begin{abstract}
Four different water/alcohol soluble polymers, poly[9,9-bis(6-( $N, N$-diethylamino)-hexyl $N$-oxide)fluorene] (PF6NO), poly[9,9-bis(6-(N,N-diethylamino)-hexyl)fluorene] (PF6N), polyethylene oxide (PEO), polyethylene imine (PEI) were used as interlayer materials in polymer light-emitting diodes (PLEDs) and polymer solar cells (PSCs) with device structures of indium tin oxide (ITO)/poly(3,4-ethylenedioxylenethiophene):poly(styrenesulphonic acid) (PEDOT:PSS)/Active Layer/Interlayer/Al (or Au), where the green light-emitting polymer poly-[2-(4-(3',7'-dimethyloctyloxy)-phenyl)-p-phenylene vinylene] (P-PPV) and photovoltaic material poly-[N-9'-hepta-decanyl-2,7-carbazole-alt-5,5-(4',7'-di-2-thienyl-2',1',3benzothiadiazole) (PCDTBT):[6,6]-phenyl C 71-butyric acid methyl ester $\left(\mathrm{PC}_{71} \mathrm{BM}\right)$ were used as the light emitting layer and light absorption layers for PLEDs and PSCs, respectively. The relationships between chemical structure and optoelectronic properties of the polymer interlayer materials were systematically investigated. Photovoltaic measurement were used to determine the built-in potential across the devices and consequently to understand the interface modification abilities of these materials. It was found that the devices based on conjugated interlayer materials exhibited larger open circuit voltages than those of devices based on non-conjugated interlayer materials, which indicates that the conjugated interlayer materials lead to lower energetic barriers for electron. Device studies showed that the conjugated interlayer materials PF6NO and PF6N exhibited much better device performance both in PLEDs and PSCs compared to the non-conjugated interlayer materials PEO and PEI, which was consistent with the photovoltaic measurement results. PLEDs studies indicated that the devices based on non-conjugated interlayer materials PEO and PEI exhibited dramatically decreased efficiencies when the interlayers' thicknesses are more than $20 \mathrm{~nm}$, while the devices based on conjugated interlayer materials PF6NO and PF6N maintained good performances. Moreover, both PLEDs and PSCs device studies indicated that the conjugated interlayer materials show a wide range of adaptability, which work well for both $\mathrm{Al}$ and $\mathrm{Au}$ electrode devices, while the non-conjugated interlayer materials only work well for the Al electrode devices. Our results indicated that besides the highly polar side chain groups, the conjugated interlayer materials' main chains also play an important role on their excellent interfacial modification ability, which lead to easily electron transporting and wide range of adaptability.

Keywords polymer light-emitting diodes; polymer solar cells; interlayer material; water/alcohol soluble polymer
\end{abstract}

\section{1 引言}

近年来，水/醇溶聚合物界面修饰材料由于在有机
光电器件上的成功应用, 得到了科学界的广泛关注 ${ }^{[1]}$. 水/醇溶聚合物界面修饰材料独特的溶解特性，使其应 用在有机光电器件上时表现出诸多优势. 首先, 可利用

\footnotetext{
*E-mail: msfhuang@scut.edu.cn

Received October 15, 2012; published December 7, 2012.
}

Project supported by the Ministry of Science and Technology (Nos. 2009CB623601 and 2009CB930604), the Natural Science Foundation of China (Nos. 21125419, 50990065, 51010003 and 51073058) and Guangdong Natural Science Foundation (No. S2012030006232).

项目受科技部 973 计划(Nos. 2009CB623601, 2009CB930604)、国家自然科学基金(Nos. 21125419, 50990065, 51010003, 51073058)以及广东省自然科学 基金(No. S2012030006232)资助. 
水/醇等环境友好的溶剂进行加工, 这非常有利于环境 保护，同时又适合工业化生产，降低成本; 其次，由于 常见的传统聚合物半导体活性层材料大多不溶于水/醇 类溶剂, 故水/醇溶聚合物界面修饰材料可通过溶液加 工的方法与传统聚合物半导体材料共同制备高效多层 器件, 同时避免不同功能层间界面互溶; 最后, 研究发 现水/醇溶聚合物界面修饰材料能够在活性层与稳定的 高功函金属或金属氧化物电极之间形成偶极作用而降 低电极功函数, 有利于电子的注入或收集，从而制备高 效稳定的有机光电器件 ${ }^{[1 \sim 4]}$. 水/醇溶聚合物界面修饰材 料从主链结构上来分, 可分为两类: 一类是主链共轭的 水/醇溶共轭聚合物材料, 如聚 [9,9-二(3'-( N,N-二甲胺 基)丙基)-2,7-芴 ]-交-2,7-(9,9-二辛基芴)](PFN), 聚二溴 化 [9,9-二(3'-((N,N-二甲基)- $N$-乙氨盐基)-丙基)2,7-芴 ${ }^{-}$ 交-1,4-苯基](PFNBr) 等; 另外一类是不具有共轭结构的 传统聚合物表面活性剂材料, 如: 聚氧化乙烯(PEO), 嵌段共聚物 F127, 聚乙烯亚胺(PEI), 聚乙氧化乙烯亚 胺(PEIE) 等 ${ }^{[2,5 \sim 12]}$. 共轭水/醇溶聚合物界面材料自从 2004 年被报道以来 ${ }^{[2,5,13]}$, 已被成功的应用至聚合物电 子器件, 实现了高效的红绿蓝及白光聚合物发光二极管

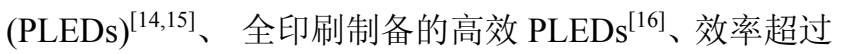

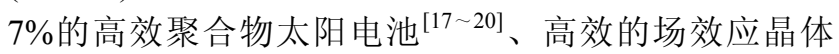
管 ${ }^{[21]}$ 以及场效应发光晶体管等 ${ }^{[22,23]}$, 显示了巨大的应用 潜力. 与此同时, 非共轭的水/醇溶聚合物界面材料也在 有机光电器件上得到了成功的应用, 实现了高效的聚合 物发光器件 ${ }^{[24,25]}$, 聚合物太阳电池 ${ }^{[12,26,27]}$ 等. 目前的研 究表明, 共轭及非共轭水/醇溶界面材料中含有的极性 基团，在它们的独特界面修饰作用中起了关键作用，可 以与金属或金属氧化物电极发生相互作用形成界面偶 极而降低电极功函数, 增强电极电子的注入与收集. 但 对于水/醇溶界面材料本身链结构, 特别是对于共轭类 与非共轭类聚合物界面材料之间的对比研究却鲜有报 道. 而深入研究材料结构与性能关系, 对于进一步深入 了解界面材料作用机制, 发展新型高效的水/醇溶聚合 物界面材料具有重要意义.

本文分别选用共轭类水/醇溶界面修饰材料聚 $[9,9-$ 二(6-(N,N-二乙氨基)-己基 $)-$ 芴 $](\mathrm{PF} 6 \mathrm{~N})$, 聚 $[9,9-$ 二 (6-( $N, N$-二乙氨基) -己基- $N$-氧化) - 芴 $](\mathrm{PF} 6 \mathrm{NO})^{[28]}$ 和非共 轭界面修饰材料 PEO, PEI 作为研究对象, 如图式 1 所 示. PF6 NO 和 PF6N 是由共轭的主链和带有强极性基团 的侧链构成: 共轭主链赋予其良好的半导体性质 (PF6NO 的最高占有轨道能级(HOMO), 最低末占有轨 道能级(LUMO)分别为 -5.50 和 $-2.62 \mathrm{eV} ;$ PF $6 \mathrm{~N}$ 的 HOMO, LUMO 分别为 -5.49 和 $-2.59 \mathrm{eV}$ ), 这也是有别 于非共轭材料的最重要的特性; 同时极性侧链又使其在 极性溶剂里具有很好的溶解性. 而 PEO 和 PEI 则完全是 由非共轭的链段构成, 强极性基团保证其在极性溶剂有 很好的溶解性, 但材料本身是不具导电性的绝缘材料.
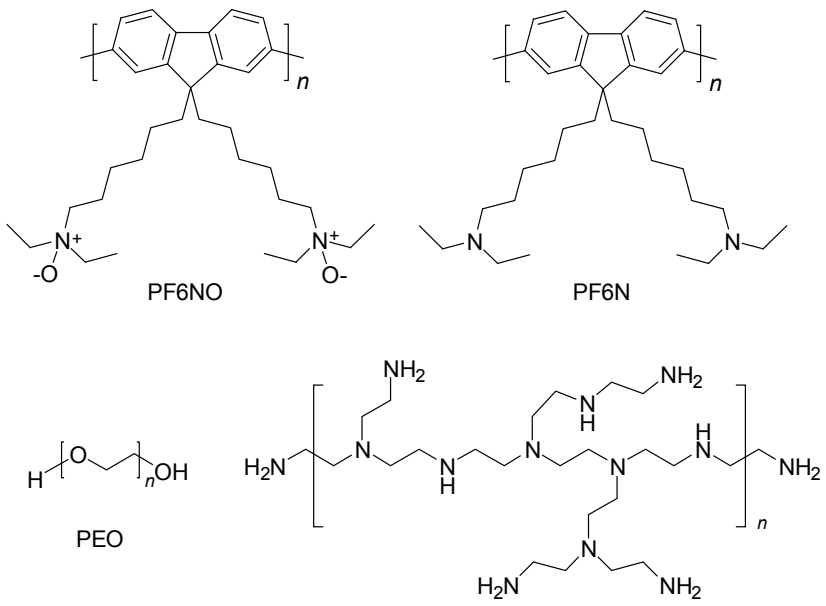

PEI

图式 1 PF6NO, PF6N, PEO 和 PEI 的化学结构

Scheme 1 Chemical structures of PF6NO, PF6N, PEO and PEI

以上所有材料均被用作界面修饰材料应用于聚合物发 光二极管器件和聚合物太阳电池器件中, 系统比较了材 料在不同器件中的性能, 研究了性能差异的原因.

\section{2 结果与讨论}

\section{1 界面材料在聚合物发光器件中的性能研究}

\section{1 .1 基于铝电极的器件性能研究}

分别以共轭与非共轭材料为电子注入层, 制得器件 结构为氧化铟锡(ITO)/聚 3,4-乙撑二氧噻吩: 聚苯乙烯 磺酸盐(PEDOT:PSS)/聚对苯撑乙烯撑共聚物(P-PPV)/电 子注入层 $(\mathrm{EIL}) / \mathrm{Al}$ 的聚合物发光二极管. 当 $\mathrm{EIL}$ 厚度 为 $10 \mathrm{~nm}$ 时, 器件的性能测试曲线如图 1,2 中所示. 未使用任何界面材料作为 EIL 时, 由于 $\mathrm{Al}$ 电极与 P-PPV 间有较大的电子注入势垒, 器件的起亮电压高达 $6.4 \mathrm{~V}$, 在 $100 \mathrm{~cd} \cdot \mathrm{m}^{-2}$ 时的流明效率仅为 $0.45 \mathrm{~cd} \cdot \mathrm{A}^{-1}$. 器件的最 大亮度和最大流明效率分别为 $421 \mathrm{~cd} \cdot \mathrm{m}^{-2}$ 和 $0.56 \mathrm{~cd} \bullet$ $\mathrm{A}^{-1}$. 为了排除界面材料所使用溶剂对器件性能的影响, 同比制备了甲醇/ $\mathrm{Al}$ 的器件. 发现相对于 $\mathrm{Al}$ 阴极的器件, 甲醇/Al 器件性能略有提升, 这有可能是由界面间残留 的甲醇分子与 $\mathrm{Al}$ 电极形成的偶极相互作用引起 ${ }^{[29]}$. 当 使用非共轭类界面材料 EIL 后, 器件的性能得到了大幅 提升: 起亮电压分别降到了 $2.8 \mathrm{~V}$ (PEO)和 $3.2 \mathrm{~V}$ (PEI), 最大亮度和最大流明效率也分别达到了 $15065 \mathrm{~cd} \cdot \mathrm{m}^{-2}$ 和 $12.91 \mathrm{~cd} \cdot \mathrm{A}^{-1}$ (PEO)以及 $12633 \mathrm{~cd} \bullet \mathrm{m}^{-2}$ 和 $9.48 \mathrm{~cd} \bullet$ $\mathrm{A}^{-1}$ (PEI). 这说明非共轭类界面材料能在一定程度上降 低 $\mathrm{Al}$ 电极的功函数, 从而起到良好的电子注入作用 ${ }^{[3]}$. 当使用共轭类界面材料 PF6N 后, 器件也表现出很好的 性能: 起亮电压降到了 $3.8 \mathrm{~V}$, 最大亮度和最大效率分 别达到 $8744 \mathrm{~cd} \cdot \mathrm{m}^{-2}$ 和 $12.91 \mathrm{~cd} \cdot \mathrm{A}^{-1}$. 当使用共轭界面材 料 PF6NO 后, 起亮电压降到 $2.8 \mathrm{~V}$, 最大亮度和最大效 率达 $21160 \mathrm{~cd} \bullet \mathrm{m}^{-2}$ 和 $18.07 \mathrm{~cd} \bullet \mathrm{A}^{-1}$, 其性能相对于基于 非共轭聚合物界面材料的器件性能有明显提高. 


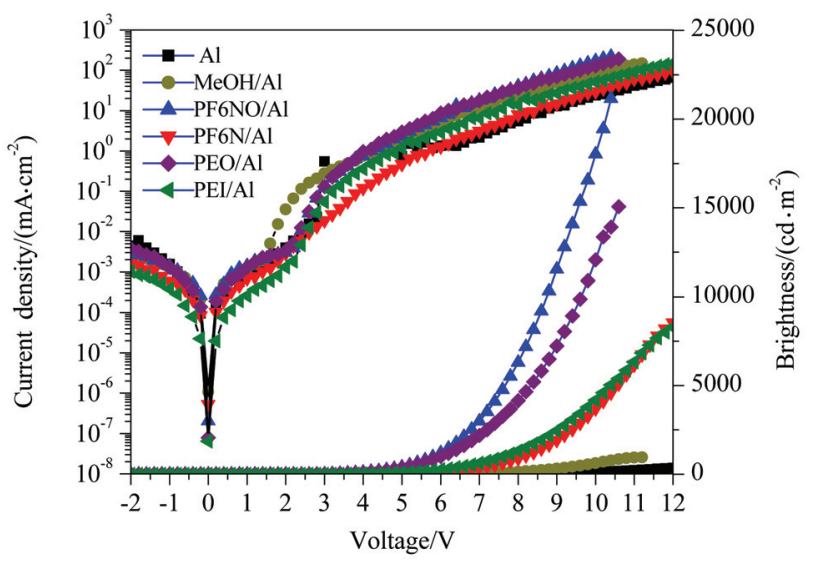

图 1 电流密度 $(J)$ 和亮度 $(L)$ 对电压 $(V)$ 的曲线

Figure 1 Current density $(J)$ and brightness $(L)$ versus voltage $(V)$ characteristics $(J-L-V)$

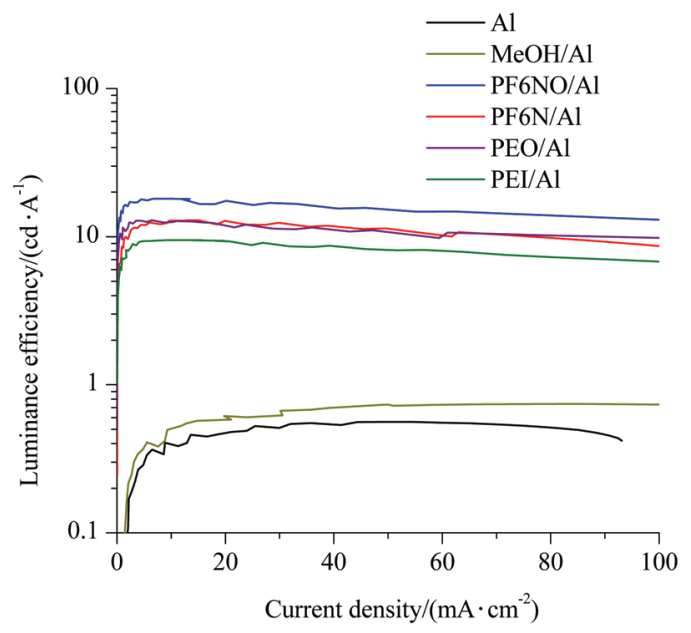

图 2 流明效率 $(L E)$ 对电流密度 $(J)$ 的曲线

Figure 2 Luminous efficiency $(L E)$ versus current density $(J)$ characteristics

光伏测试被广泛用来测定器件内部的内建电势从 而了解界面层的作用机理, 器件开路电压的大小可以间 接反映不同界面材料电子注入/界面修饰能力的强 弱 ${ }^{[2,28]}$. 如图 3 所示, 纯铝阴极和甲醇/Al 阴极的开路电 压分别为 1.38 和 $1.36 \mathrm{~V}$. 当使用了界面修饰材料 PEO 和 PF6N 后, 开路电压分别增加到 1.56 和 $1.60 \mathrm{~V}$, 这表 明电子的注入势垒被降低了, 电子可以更容易的从阴极 注入至发光层, 从而使器件内的载流子注入与复合更加 平衡. 因此, 器件性能相对于无界面修饰材料的器件得 到了大幅的提高. 当使用 PF6NO 作为界面修饰材料后, 开路电压进一步提高到了 $1.86 \mathrm{~V}$. 这说明 PF6NO/Al 阴 极的电子注入势垒相对于 $\mathrm{PEO} / \mathrm{Al}$ 和 PF6N/Al 阴极的势 垒有进一步降低. 因此, PF6NO 将更有利于电子的注入, 从而获得更平衡的载流子(电子与空穴)注入以及更高的 器件性能. 在误差范围内, 器件开路电压(PF6NO/Al>
$\mathrm{PF} 6 \mathrm{~N} / \mathrm{Al} \approx \mathrm{PEO} / \mathrm{Al}>\mathrm{PEI} / \mathrm{Al} \approx \mathrm{MeOH} / \mathrm{Al} \approx \mathrm{Al})$ 与器件性 能结果保持了很好的一致性( $\mathrm{PF} 6 \mathrm{NO} / \mathrm{Al}>\mathrm{PF} 6 \mathrm{~N} / \mathrm{Al} \approx$ $\mathrm{PEO} / \mathrm{Al}>\mathrm{PEI} / \mathrm{Al}>\mathrm{MeOH} / \mathrm{Al} \approx \mathrm{Al})$.

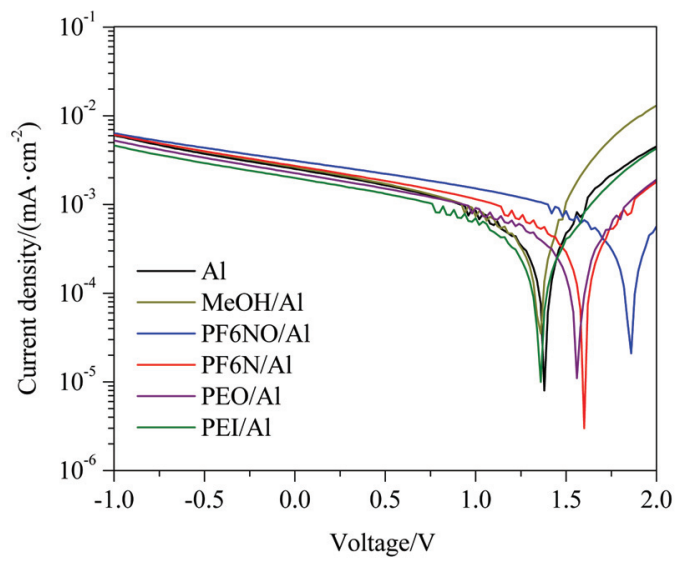

图 $3 \mathrm{Al}$ 阴极器件的光伏特性曲线

Figure 3 Photovoltaic characteristics of these devices with Al cathode

需要注意的是, $\mathrm{PEI} / \mathrm{Al}$ 的开路电压接近 $\mathrm{MeOH} / \mathrm{Al}$ 和 $\mathrm{Al}$, 但器件性能却优于 $\mathrm{MeOH} / \mathrm{Al}$ 和 $\mathrm{Al}$. 这可能是由 于 PEI 的熔点比较低, 在进行光伏测试时, 测试过程中 的温度升高引起 PEI 融化, 从而导致测试结果与实际器 件性能出现差异.

为了研究界面材料厚度对器件性能的影响, 制备了 不同厚度界面材料的发光二极管器件. 表 1 列出了不同 界面层厚度 (从 $\approx 5 \mathrm{~nm}$ 变化到 $\approx 20 \mathrm{~nm}$ )下相关器件的器 件性能. 结果表明, 共轭类的界面材料 PF6N 和 PF6NO 在 $\approx 20 \mathrm{~nm}$ 的厚度条件下，仍然保持着最大流明效率 12.71 和 $15.34 \mathrm{~cd} \cdot \mathrm{A}^{-1}$, 而非共轭类的界面材料 PEO 和 PEI 的器件性能降低明显, 最大流明效率分别降到了 3.19 和 $8.32 \mathrm{~cd} \cdot \mathrm{A}^{-1}$. 这是因为共轭类界面材料共轭的主 链使材料具有了良好的电子传输性能; 而非共轭界面材 料属于绝缘体, 电子很难通过 $\approx 20 \mathrm{~nm}$ 厚度的绝缘层传 输到发光层内复合发光, 最终导致器件性能大幅降低. 当界面层厚度减少至 $\approx 5 \mathrm{~nm}$ 时, 电子可以通过遂穿机 制成功的穿过绝缘材料实现注入 ${ }^{[30,31]}$. 因此相对于 $\approx 20$ $\mathrm{nm}$ 厚度的器件, 非共轭界面材料在 $\approx 5 \mathrm{~nm}$ 厚度条件下 保持了较好的器件性能, 其中基于 PEI 器件的最大流明 效率为 $10.33 \mathrm{~cd} \cdot \mathrm{A}^{-1}$, 基于 $\mathrm{PEO}$ 器件的最大流明效率为 $4.93 \mathrm{~cd} \cdot \mathrm{A}^{-1}$; 而共轭类的界面材料则在大的厚度范围内 均保持很好的器件性能. 需要注意是 $\mathrm{PF} 6 \mathrm{~N}$ 在 $\approx 5 \mathrm{~nm}$ 条 件下, 器件性能很差 $\left(L E_{\max }=0.45 \mathrm{~cd} \cdot \mathrm{A}^{-1}\right)$, 这可能是由 于 PF6N 在很薄的条件下无法形成有效的界面偶极, 最 终导致电子无法高效的注入.

\subsection{2 基于金电极的器件性能研究}

之前的研究结果表明，水/醇溶共轭聚合物界面修 饰材料对金这一类十分稳定的金属也具有优良的界面 修饰作用，能明显的增强电子从金电极向活性层的注 
表 1 用不同界面材料修饰不同金属阴极的聚合物发光二极管器件性能比较

Table 1 Device performances of PLEDs using different EIL/Al (Au) as a cathode in device configuration ITO/PEDOT/P-PPV/EIL/metals

\begin{tabular}{|c|c|c|c|c|c|}
\hline Cathode & $V_{\text {on }} / \mathrm{V}$ & $L E^{a} /\left(\mathrm{cd} \cdot \mathrm{A}^{-1}\right)$ & $L E^{b} /\left(\mathrm{cd} \cdot \mathrm{A}^{-1}\right)$ & $L_{\max } /\left(\mathrm{cd} \cdot \mathrm{m}^{-2}\right)$ & $L E_{\max } /\left(\mathrm{cd} \cdot \mathrm{A}^{-1}\right)$ \\
\hline $\mathrm{Al}$ & 6.4 & 0.45 & - & 421 & 0.56 \\
\hline $\mathrm{MeOH} / \mathrm{Al}$ & 4.8 & 0.48 & - & 950 & 0.74 \\
\hline $\mathrm{PF} 6 \mathrm{NO}(5 \mathrm{~nm}) / \mathrm{Al}$ & 3.4 & 6.13 & 8.37 & 11062 & 9.86 \\
\hline PF6NO(12 nm)/Al & 2.8 & 14.89 & 17.56 & 21160 & 18.07 \\
\hline $\mathrm{PF} 6 \mathrm{NO}(22 \mathrm{~nm}) / \mathrm{Al}$ & 3.0 & 12.99 & 15.34 & 8282 & 15.34 \\
\hline PF6N(5 nm)/Al & 4.8 & 0.36 & - & 735 & 0.45 \\
\hline $\mathrm{PF} 6 \mathrm{~N}(12 \mathrm{~nm}) / \mathrm{Al}$ & 3.8 & 8.39 & 11.38 & 8744 & 12.91 \\
\hline PF6N(24 nm)/Al & 4.8 & 10.67 & 11.32 & 5269 & 12.71 \\
\hline $\mathrm{PEO}(5 \mathrm{~nm}) / \mathrm{Al}$ & 3.0 & 4.78 & 3.53 & 3140 & 4.93 \\
\hline $\mathrm{PEO}(10 \mathrm{~nm}) / \mathrm{Al}$ & 2.8 & 11.53 & 11.46 & 15065 & 12.91 \\
\hline $\mathrm{PEO}(25 \mathrm{~nm}) / \mathrm{Al}$ & 4.8 & 2.47 & - & 426 & 3.19 \\
\hline $\mathrm{PEI}(6 \mathrm{~nm}) / \mathrm{Al}$ & 3.2 & 7.39 & 10.26 & 14073 & 10.33 \\
\hline $\operatorname{PEI}(9$ nm)/Al & 3.2 & 6.24 & 8.67 & 12633 & 9.48 \\
\hline $\operatorname{PEI}(21 \mathrm{~nm}) / \mathrm{Al}$ & 3.8 & 5.32 & 7.93 & 12543 & 8.32 \\
\hline $\mathrm{Au}$ & 11.2 & - & - & 16 & 0.01 \\
\hline $\mathrm{MeOH} / \mathrm{Au}$ & 11.4 & - & - & 19 & 0.01 \\
\hline $\mathrm{PF} 6 \mathrm{NO}(5 \mathrm{~nm}) / \mathrm{Au}$ & 5.8 & 0.46 & - & 253 & 0.51 \\
\hline $\operatorname{PF} 6 \mathrm{NO}(12 \mathrm{~nm}) / \mathrm{Au}$ & 3.4 & 9.14 & 9.75 & 5278 & 10.58 \\
\hline $\mathrm{PF} 6 \mathrm{NO}(22 \mathrm{~nm}) / \mathrm{Au}$ & 3.6 & 9.39 & 9.61 & 3942 & 10.56 \\
\hline $\mathrm{PF} 6 \mathrm{~N}(5 \mathrm{~nm}) / \mathrm{Au}$ & 10.2 & - & - & 21 & 0.03 \\
\hline $\operatorname{PF} 6 \mathrm{~N}(12 \mathrm{~nm}) / \mathrm{Au}$ & 5.6 & 1.16 & - & 768 & 2.34 \\
\hline $\mathrm{PF} 6 \mathrm{~N}(24 \mathrm{~nm}) / \mathrm{Au}$ & 4.8 & 6.83 & 8.87 & 2674 & 8.90 \\
\hline $\mathrm{PEO}(5 \mathrm{~nm}) / \mathrm{Au}$ & 11.6 & - & - & 32 & 0.01 \\
\hline $\mathrm{PEO}(10 \mathrm{~nm}) / \mathrm{Au}$ & 13.4 & - & - & 72 & 0.02 \\
\hline $\mathrm{PEO}(25 \mathrm{~nm}) / \mathrm{Au}$ & 12.6 & - & - & 64 & 0.56 \\
\hline $\operatorname{PEI}(6 \mathrm{~nm}) / \mathrm{Au}$ & 8.8 & 0.81 & - & 609 & 1.37 \\
\hline $\operatorname{PEI}(9 \mathrm{~nm}) / \mathrm{Au}$ & 9.4 & 0.23 & - & 226 & 0.23 \\
\hline $\operatorname{PEI}(21 \mathrm{~nm}) / \mathrm{Au}$ & 10.0 & 0.28 & - & 282 & 0.29 \\
\hline
\end{tabular}

${ }^{a}$ Device performance at $100 \mathrm{~cd} \cdot \mathrm{m}^{-2} ;{ }^{b}$ Device performance at $1000 \mathrm{~cd} \bullet \mathrm{m}^{-2}$.

$\lambda^{[14]}$. 为了进一步研究非共轭与共轭类界面材料对高 功函数电极的作用差异, 制备了以金为电极的发光二极 管器件. 实验结果如图 4, 5, 表 1 所示. 在金电极器件 中, 金电极与发光层之间存在着极高的势垒, 电子很难 从金电极注入到发光层中, 因此效率极低; 而使用甲醇 处理过的器件, 其性能也并没有任何提升, 这说明对于 金这一类超高功函数的金属电极, 残留的甲醇无法在金 与发光层界面形成有效的偶极作用. 而共轭类材料 PF6NO, PF6N 均表现出很好的电子注入/传输性能. 当 厚度为 $\sim 10 \mathrm{~nm}$ 时, PF6N 和 PF6NO 器件的起亮电压分 别为 3.4 和 $5.6 \mathrm{~V}$, 比纯 $\mathrm{Au}$ 电极器件 $\left(V_{\mathrm{on}}=11.2 \mathrm{~V}\right)$ 和甲 醇 $/ \mathrm{Au}$ 器件 $\left(V_{\mathrm{on}}=11.4 \mathrm{~V}\right)$ 的起亮电压低了近 $6 \mathrm{~V}$; 同时基 于共轭界面材料 PF6N 和 PF6NO 器件的最大流明效率 也分别达到了 $2.34 \mathrm{~cd} \cdot \mathrm{A}^{-1}$ 和 $10.58 \mathrm{~cd} \cdot \mathrm{A}^{-1}$, 这比纯 $\mathrm{Au}$ 器件 $\left(L E_{\max }=0.01 \mathrm{~cd} \cdot \mathrm{A}^{-1}\right)$ 和甲醇 $/ \mathrm{Au}$ 器件 $\left(L E_{\max }=0.01\right.$ $\left.\mathrm{cd} \cdot \mathrm{A}^{-1}\right)$ 的最大流明效率提高了 100 倍以上. 这说明共轭

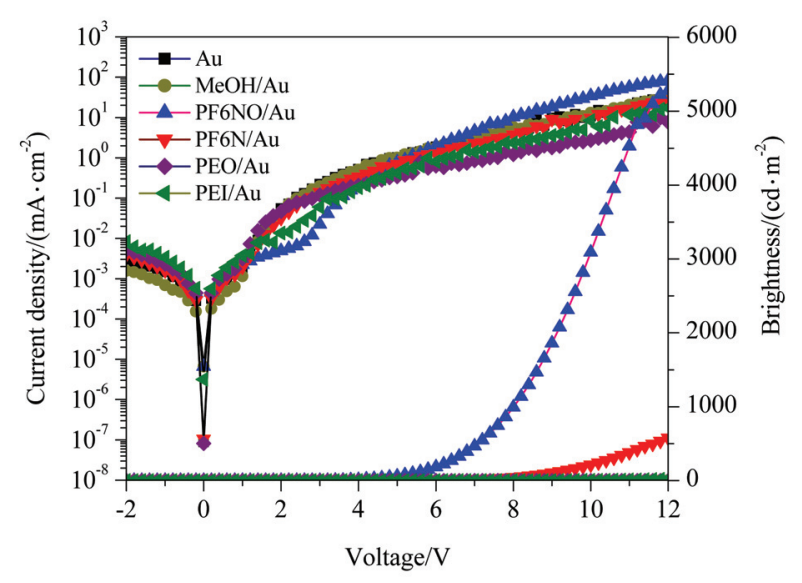

图 4 电流密度 $(J)$ 和亮度 $(L)$ 对电压 $(V)$ 的曲线

Figure 4 Current density $(J)$ and brightness $(L)$ versus voltage $(V)$ characteristics $(J-L-V)$ 


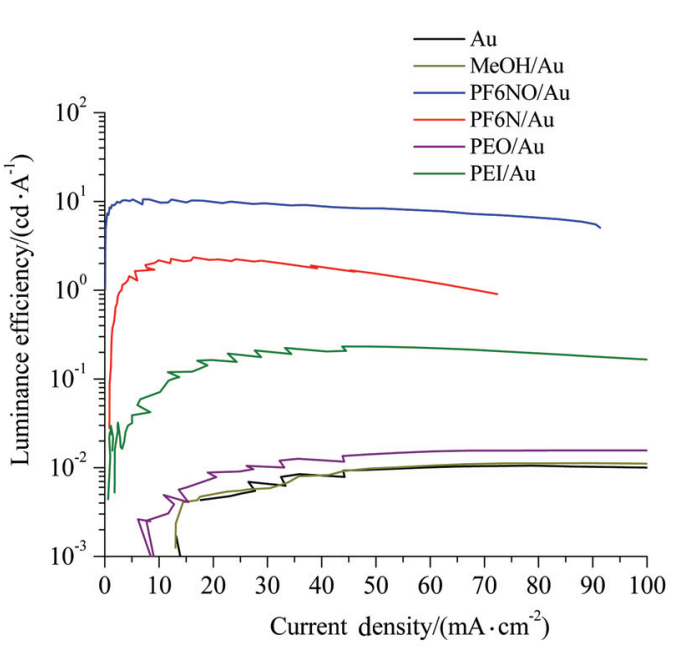

图 5 流明效率 $(L E)$ 对电流密度 $(J)$ 的曲线

Figure 5 Luminous efficiency $(L E)$ versus current density $(J)$ characteristics

类界面材料在发光层与金电极间形成的偶极能够有效 地降低金电极的功函数, 使得电子注入的势垒降低, 从 而大幅提高器件性能. 而基于非共轭类界面材料 PEO, PEI 的器件性能很差(PEO 器件的 $V_{\mathrm{on}}=13.4 \mathrm{~V}, L E_{\max }=$ $0.02 \mathrm{~cd} \cdot \mathrm{A}^{-1}$; PEI 器件的 $V_{\mathrm{on}}=9.4 \mathrm{~V}, L E_{\max }=0.23 \mathrm{~cd} \bullet$ $\left.\mathrm{A}^{-1}\right)$, 器件性能未得到大的提升. 这说明, 对于高功函 数的金电极, 共轭类界面材料的界面修饰性能明显优于 非共轭类界面材料.

\section{2 界面材料在聚合物太阳电池中的性能研究}

\subsection{1 基于铝电极的器件性能研究}

为了研究不同界面材料在聚合物太阳电池中的界 面修饰能力, 以聚 $[N-9$ "-十七烷基-2,7-咔唑-交-5,5-(4',7'二-2-噻吩基-2',1',3'-苯并噻二唑)](PCDTBT):[6,6]-苯 基-C71-丁酸甲酯 $\left(\mathrm{PC}_{71} \mathrm{BM}\right)$ 为活性层, 制备了器件结构 为 $\mathrm{ITO} / \mathrm{PEDOT}(40 \mathrm{~nm}) / \mathrm{PCDTBT}: \mathrm{PC}_{71} \mathrm{BM}(80 \mathrm{~nm}) /$ 电子 传输层 $\operatorname{ETL}(5 \mathrm{~nm}) / \mathrm{Al}(100 \mathrm{~nm})$ 的太阳电池器件. 其电 流电压特性曲线如图 6 和表 2 所示. 当不用任何阴极界 面材料时, 纯铝阴极的器件开路电压为 $0.83 \mathrm{~V}$, 短路电 流为 $7.03 \mathrm{~mA} \cdot \mathrm{cm}^{-2}$, 填充因子为 $56.86 \%$, 效率达到了 4.74\%. 当用甲醇处理活性层后, 器件的短路电流基本 保持不变, 为 $7.00 \mathrm{~mA} \cdot \mathrm{cm}^{-2}$, 开路电压略有提高, 达到 了 $0.87 \mathrm{~V}$, 这说明极性溶剂处理对太阳电池器件性能有 一定影响，与文献报道一致 ${ }^{[10]}$. 当使用共轭类界面材料 PF6NO 和 PF6N 后, 器件的短路电流略有变化, 但是开 路电压 $V_{\mathrm{oc}}$ 却大幅的提高至 $0.93 \mathrm{~V}$ 从而使器件性能得到 明显提升. 基于非共轭类界面材料 PEO 和 PEI 的器件, $V_{\mathrm{oc}}$ 也得到了提高. 这主要是因为这些界面修饰材料在 金属阴极与活性层之间形成的偶极可以起到增强器件 内建电场的作用, 最终使得器件的 $V_{\mathrm{oc}}$ 增加 ${ }^{[17,26,32]}$. 但 是, 相对应其它界面材料修饰的器件, 基于 PEO 的器件 短路电流较低, 这可能是因为 PEO 的导电能力较差, 使 得器件的串联电阻升高, 阻碍了电子在阴极的收集和传
输，从而使得电流降低.

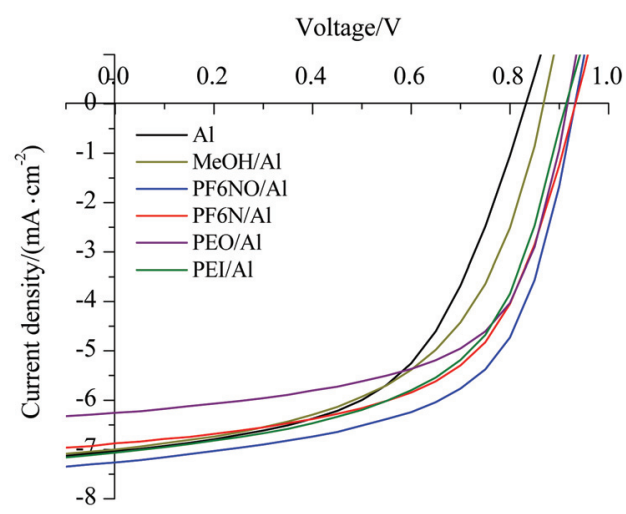

图 6 铝电极器件的电流 $(J)$-电压 $(V)$ 特性曲线

Figure 6 Current density $(J)$ versus voltage $(V)$ characteristic of devices with $\mathrm{Al}$ cathode

表 2 用不同界面材料修饰的不同金属阴极的聚合物太阳电池器件性 能比较

Table 2 Photovoltaic performances of PSCs using different ETL/Al $\mathrm{Au})$ as a cathode in device configuration ITO/PEDOT/PCDTBT: $\mathrm{PC}_{71} \mathrm{BM}(1: 4) / \mathrm{ETL} /$ metals

\begin{tabular}{lcccc}
\hline \multicolumn{1}{c}{ Cathode } & $J_{\mathrm{sc}}\left(\mathrm{mA} \cdot \mathrm{cm}^{-2}\right)$ & $V_{\mathrm{oc}} / \mathrm{V}$ & $\mathrm{FF} / \%$ & $\mathrm{PCE} / \%$ \\
\hline $\mathrm{Al}$ & 7.03 & 0.83 & 56.86 & 4.74 \\
$\mathrm{MeOH} / \mathrm{Al}$ & 7.00 & 0.87 & 56.32 & 4.90 \\
$\mathrm{PF} 6 \mathrm{NO} / \mathrm{Al}$ & 7.27 & 0.93 & 62.84 & 6.07 \\
$\mathrm{PF} 6 \mathrm{~N} / \mathrm{Al}$ & 6.87 & 0.93 & 61.13 & 5.58 \\
$\mathrm{PEO} / \mathrm{Al}$ & 6.25 & 0.92 & 60.26 & 4.95 \\
$\mathrm{PEI} / \mathrm{Al}$ & 7.06 & 0.92 & 55.93 & 5.19 \\
$\mathrm{Au}$ & 5.64 & 0.75 & 48.98 & 2.96 \\
$\mathrm{MeOH} / \mathrm{Au}$ & 5.83 & 0.80 & 43.07 & 2.87 \\
$\mathrm{PF} 6 \mathrm{NO} / \mathrm{Au}$ & 5.63 & 0.91 & 65.17 & 4.77 \\
$\mathrm{PF} 6 \mathrm{~N} / \mathrm{Au}$ & 5.39 & 0.90 & 63.06 & 4.37 \\
$\mathrm{PEO} / \mathrm{Au}$ & 5.25 & 0.52 & 42.2 & 1.83 \\
$\mathrm{PEI} / \mathrm{Au}$ & 5.43 & 0.88 & 51.7 & 3.92 \\
\hline
\end{tabular}

\subsection{2 基于金电极的器件性能研究}

为了研究不同界面材料在聚合物太阳电池中对不 同金属电极的修饰能力, 制备了以金为电极的电池器 件, 结构与铝电极器件类似. 如图 7 和表 2 所示. 纯金电 极的器件, 其短路电流为 $5.64 \mathrm{~mA} \cdot \mathrm{cm}^{-2}$, 开路电压为 $0.75 \mathrm{~V}$, 填充因子为 $48.98 \%$, 效率仅有 $2.96 \%$. 这是因 为金的功函数高, 无法与活性层形成欧姆接触，电子向 阴极传输时需要越过很高的能垒, 从而导致器件电流很 低; 同时, 因为金的功函数较高, 导致器件的内建电场 较小 ${ }^{[33]}$, 使器件的开路电压较低, 最终影响器件的性 能. 而用甲醇处理的器件, 器件性能无明显变化, 这说 明甲醇对高功函数的金属电极修饰作用十分有限, 不能 降低金属的功函数, 起到增强内建电场的作用, 这与在 发光器件中观测到的结果一致. 当使用界面材料 PF6NO, PF6N 和 PEI 后, 由于界面层可在活性层与金电 


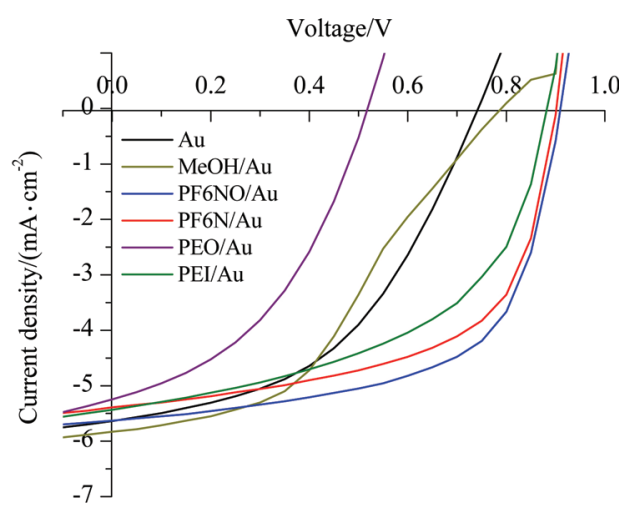

图 7 金电极器件的电流 $(J)$-电压 $(V)$ 特性曲线

Figure 7 Current density $(J)$ versus voltage $(V)$ characteristic of devices with $\mathrm{Au}$ cathode

极之间形成有效的偶极作用, 增强器件的内建电场, 从 而使相关器件的 $V_{\mathrm{oc}}$ 均提高到 $0.9 \mathrm{~V}$ 左右, 大幅提高了相 关器件的效率(表 2). 值得注意的是, PEO 修饰的金电极 器件, 其开路电压只有 $0.52 \mathrm{~V}$, 甚至低于纯金电极器件 的开路电压, 说明 PEO 对 $\mathrm{Au}$ 电极无法起到有效的修饰 作用. 同时, PEO 的引入还增加了器件的串联电阻, 阻 碍了电荷的收集, 使得器件电流降低. 然而, 在以 $\mathrm{Al}$ 为 电极的器件中, PEO 修饰作用明显, 器件的开路电压达 到了 $0.92 \mathrm{~V}$. 这可能是因为蒸镀的铝原子可以和 PEO 上 的氧原子配合作用使得靠近 PEO 的活性层被 $\mathrm{n}$-型掺杂, 而金原子活性低, 无此反应特性 ${ }^{[34]}$. 以上结果表明, 共 轭类水/醇溶界面材料的共轭主链和极性侧链基团使其 具有良好的电子收集和传输能力, 这使得其应用在聚合 物太阳电池中时, 表现出优异的界面修饰作用. 而非共 轭类水/醇溶界面材料则受限于其饱和的主链结构, 电 子传输能力较差, 应用在太阳电池的界面修饰方面, 性 能也差于共轭类界面材料.

\section{3 结论}

通过系列不同结构的器件系统研究了共轭类 (PF6NO, PF6N)和非共轭类(PEO, PEI)水/醇溶界面修饰 材料的性能. 结果表明, 在聚合物发光二极管中, 非共 轭类界面材料对铝电极表现出了有效的电子注入/传输 能力, 对金电极则没有明显的作用. 而共轭类界面材料 对铝, 金电极均表现出了优异的电子注入/传输性能, 使 得金属阴极和发光层之间电子注入的势垒得到了大幅 的降低, 电子更容易注入到器件内, 从而使得载流子注 入更加平衡, 大幅提高相关器件的性能. 同时共轭类界 面材料良好的半导体性能使其在大的厚度范围内都有 良好的界面修饰能力. 在聚合物太阳电池中, 非共轭与 共轭类材料可以在金属电极和有机层之间形成界面偶 极, 增加器件的内建电场, 降低金属电极功函数, 提高 器件性能. 研究表明共轭与非共轭材料对铝电极都有一 定界面修饰作用, 但基于共轭类界面材料性能更为优
异，与在聚合物发光二极管中观测到的结果是一致的. 这一系列的研究结果表明: 共轭类界面修饰材料 (PF6NO，PF6N) 较之于非共轭类界面修饰材料(PEO, PEI), 有更好的电子注入/传输能力, 特别是在高功函金 属金电极器件中表现更为明显, 说明除材料所含极性基 团外, 界面材料本身的半导体性能对其界面修饰能力也 有着重要影响.

\section{4 实验部分}

\section{1 聚合物发光二极管的制备}

将浌射有 ITO 的玻璃基片依次经过去离子水，丙 酮, 无泡洗液, 去离子水和异丙醇超声洗涤后放于真空 烘箱内干燥过夜. 干燥后的 ITO 玻璃基片经过 $4 \mathrm{~min}$ $\mathrm{O}_{2}$-plasma 处理后, 旋涂一层 $40 \mathrm{~nm}$ 厚的 PEDOT:PSS (Baytron P4083, Bayer AG), 并于 $150{ }^{\circ} \mathrm{C}$ 加热处理 20 min. 之后, 将发光材料 P-PPV(广州研剑光电材料科技 有限公司)的二甲苯溶液旋涂于 PEDOT 层上，制得 90 $\mathrm{nm}$ 厚的发光层. 电子注入/传输材料 $\mathrm{PF}^{2} \mathrm{~N}^{[28]}$, PF6NO ${ }^{[28]}$, PEO (aldrich, $M_{\mathrm{w}}$ 10000), PEI (aldrich, branched, $M_{\mathrm{w}} \approx 25000$ )分别溶于甲醇, 通过调节浓度和 旋涂速度制得厚度在 $5 \sim 25 \mathrm{~nm}$ 范围内的膜. 将旋涂了 有机层的玻璃基片转入高真空蒸镀仓内，通过真空热蒸 镀分别蒸镀铝, 金电极. 器件面积通过掩模板调控在 $0.16 \mathrm{~cm}^{2}$. 蒸镀了金属电极的器件, 用环氧树脂和盖玻 片, 通过紫外光固化进行包封.

\section{2 聚合物太阳电池的制备}

将溅射有 ITO 的玻璃基片依次经过去离子水，丙 酮, 无泡洗液, 去离子水和异丙醇超声洗涤后放于真空 烘箱内干燥过夜. 干燥后的 ITO 玻璃基片经过 $4 \mathrm{~min}$ $\mathrm{O}_{2}$-plasma 处理后, 旋涂一层 $40 \mathrm{~nm}$ 厚的 PEDOT:PSS (Baytron P4083, Bayer AG), 并于 $150{ }^{\circ} \mathrm{C}$ 加热处理 20 $\min$ 之后, 将光活性材料 PCDTBT (1-material):PC71BM (aldrich) $(1: 4)$ 的氯苯:二氯苯 $(1: 3)$ 溶液旋涂于 PEDOT 层上, 制得 $90 \mathrm{~nm}$ 厚的活性层. 电子注入/传输材料 PF6N, PF6NO, PEO, PEI 分别溶于甲醇, 通过调节浓度 和旋涂速度制得厚度在 $5 \mathrm{~nm}$ 左右的膜. 将旋涂了有机 层的玻璃基片转入高真空蒸镀仓内，通过真空热蒸镀分 别蒸镀铝、金电极. 器件面积通过掩模板调控在 0.16 $\mathrm{cm}^{2}$. 蒸镀了金属电极的器件用环氧树脂和盖玻片通过 紫外光固化进行包封.

\section{3 有机光电器件的性能测试}

界面层厚度通过椭圆偏振光谱仪(Auto SE Spectroscopic Ellipsometer, HORIBA Jobin Yvon)测得. 聚合物 发光二极管器件的电流密度 $(J)$-电压 $(V)$-亮度 $(L)$ 数据通 过 keithley 236 电源和校正的硅光二极管测得. 聚合物 太阳电池的光伏特性曲线以及聚合物发光二极管的内 建电场均通过 AM 1.5G 模拟太阳光灯 (Oriel model 
91192)测得.

\section{References}

[1] Huang, F.; Wu, H.; Cao, Y. Chem. Soc. Rev. 2010, 39, 2500

[2] Wu, H.; Huang, F.; Mo, Y.; Yang, W.; Wang, D.; Peng, J.; Cao, Y. Adv. Mater. 2004, 16, 1826.

[3] Niu, Y.-H.; Ma, H.; Xu, Q.; Jen, A. K.-Y. Appl. Phys. Lett. 2005, 86, 083504.

[4] Zhong, C.; Duan, C.; Huang, F.; Wu, H.; Cao, Y. Chem. Mater. 2011, 23, 326.

[5] Huang, F.; Wu, H.; Wang, D.; Yang, W.; Cao, Y. Chem. Mater. 2004, 16, 708

[6] Guo, T.-F.; Yang, F.-S.; Tsai, Z.-J.; Wen, T.-C.; Hsieh, S.-N.; Fu, Y.-S. Appl. Phys. Lett. 2005, 87, 013504

[7] Niu, X.; Qin, C.; Zhang, B.; Yang, J.; Xie, Z.; Cheng, Y.; Wang, L. Appl. Phys. Lett. 2007, 90, 203513.

[8] Xiong, T.; Wang, F.; Qiao, X.; Ma, D. Appl. Phys. Lett. 2008, 93, 123310.

[9] Lu, H. H.; Ma, Y. S.; Yang, N. J.; Lin, G. H.; Wu, Y. C.; Chen, S. A. J. Am. Chem. Soc. 2011, 133, 9634.

[10] Seo, J. H.; Gutacker, A.; Sun, Y.; Wu, H.; Huang, F.; Cao, Y.; Scherf, U.; Heeger, A. J.; Bazan, G. C. J. Am. Chem. Soc. 2011, 133, 8416.

[11] Zhang, K.; Liu, S.; Guan, X.; Duan, C.; Zhang, J.; Zhong, C.; Wang, L.; Huang, F.; Cao, Y. Sci. China Chem. 2012, 55, 766.

[12] Zhou, Y.; Fuentes-Hernandez, C.; Shim, J.; Meyer, J.; Giordano, A. J.; Li, H.; Winget, P.; Papadopoulos, T.; Cheun, H.; Kim, J.; Fenoll, M.; Dindar, A.; Haske, W.; Najafabadi, E.; Khan, T. M.; Sojoudi, H.; Barlow, S.; Graham, S.; Bredas, J.-L.; Marder, S. R.; Kahn, A.; Kippelen, B. Science 2012, 336, 327.

[13] Huang, F.; Hou, L.; Wu, H.; Wang, X.; Shen, H.; Cao, W.; Yang, W.; Cao, Y. J. Am. Chem. Soc. 2004, 126, 9845.

[14] Wu, H.; Huang, F.; Peng, J.; Cao, Y. Org. Electron. 2005, 6, 118.

[15] Huang, F.; Shih, P.-I.; Shu, C.-F.; Chi, Y.; Jen, A. K.-Y. Adv. Mater. 2009, 21,361
[16] Zeng, W. J.; Wu, H. B.; Zhang, C.; Huang, F.; Peng, J. B.; Yang, W.; Cao, Y. Adv. Mater. 2007, 19, 810.

[17] He, Z.; Zhong, C.; Huang, X.; Wong, W. Y.; Wu, H.; Chen, L.; Su, S.; Cao, Y. Adv Mater 2011, 23, 4636.

[18] Yang, T.; Wang, M.; Duan, C.; Hu, X.; Huang, L.; Peng, J.; Huang, F.; Gong, X. Energy Environ. Sci. 2012, 5, 8208.

[19] He, Z.; Zhong, C.; Su, S.; Xu, M.; Wu, H.; Cao, Y. Nat. Photon. 2012, 6, 593 .

[20] Liao, S. H.; Li, Y. L.; Jen, T. H.; Cheng, Y. S.; Chen, S. A. J. Am. Chem. Soc. 2012, 134, 14271.

[21] Lan, L.; Peng, J.; Sun, M.; Zhou, J.; Zou, J.; Wang, J.; Cao, Y. Org Electron. 2009, 10,346.

[22] Seo, J. H.; Namdas, E. B.; Gutacker, A.; Heeger, A. J.; Bazan, G. C. Adv. Funct. Mater. 2011, 21, 3667.

[23] Hsu, B. B.; Duan, C.; Namdas, E. B.; Gutacker, A.; Yuen, J. D.; Huang, F.; Cao, Y.; Bazan, G. C.; Samuel, I. D. W.; Heeger, A. J. Adv. Mater. 2012, 24, 1171

[24] Hsiao, C. C.; Hsiao, A. E.; Chen, S. A. Adv. Mater. 2008, $20,1982$.

[25] Chen, J.; Shi, C.; Fu, Q.; Zhao, F.; Hu, Y.; Feng, Y.; Ma, D. J. Mater. Chem. 2012, 22, 5164.

[26] Zhang, F.; Ceder, M.; Inganäs, O. Adv. Mater. 2007, 19, 1835.

[27] Kang, H.; Hong, S.; Lee, J.; Lee, K. Adv. Mater. 2012, 24, 3005

[28] Guan, X.; Zhang, K.; Huang, F.; Bazan, G. C.; Cao, Y. Adv. Funct. Mater. 2012, 22, 2846.

[29] Wang, Q.; Zhou, Y.; Zheng, H.; Shi, J.; Li, C.; Su, C. Q.; Wang, L.; Luo, C.; Hu, D.; Pei, J.; Wang, J.; Peng, J.; Cao, Y. Org. Electron. 2011, 12, 1858 .

[30] Shewchun, J.; Dubow, J.; Myszkowski, A.; Singh, R. J. Appl. Phys. 1978, 49, 855.

[31] Shewchun, J.; Singh, R.; Green, M. A. J. Appl. Phys. 1977, 48, 765.

[32] Na, S.-I.; Kim, T.-S.; Oh, S.-H.; Kim, J.; Kim, S.-S.; Kim, D.-Y. Appl. Phys. Lett. 2010, 97, 223305.

[33] Brabec, C. J.; Cravino, A.; Meissner, D.; Sariciftci, N. S.; Fromherz, T.; Rispens, M. T.; Sanchez, L.; Hummelen, J. C. Adv. Funct. Mater. 2001, 11, 374.

[34] Niu, Y.-H.; Jen, A. K.-Y.; Shu, C.-F. J. Phys. Chem. B 2006, 110 6010

(Zhao, X.) 